\title{
A journey through the CSD in celebration of Richard E. Marsh
}

\author{
Suzanna C. Ward ${ }^{1}$, Amy A. Sarjeant ${ }^{1}$
}

${ }^{1}$ The Cambridge Crystallographic Data Centre, 12 Union Road, Cambridge CB2 1EZ, UK

The Cambridge Structural Database (CSD) is the world's repository for organic and metal-organic structures. With well over 900,000 entries, the CSD has representative structures from every crystal system, every space group and every corner of the periodic table. The CSD is also home to a significant number of entries from Richard E. Marsh, many of which were a direct result of his detective work in identifying incorrect crystal structures.

This presentation will focus on the tremendous contribution Richard made to crystallography, and thus to the CSD, from the authoring of structures to the identification of errors in structural data which led to the development of numerous validation tools. As curators of a recognized repository for experimental structural data, we will then go on to explore the role we play in ensuring the quality and validity of the data entrusted to us. The presentation will touch on our longstanding efforts to certify the correctness of data from deposition to curation and the steps we take in maintaining a trusted high-quality database. Finally, we will discuss the steps we are taking to help identify fraudulent data, help users of the CSD assess the quality of the structures therein, and what more we could do to help future crystallographers avoid being "Marshed".

Memorial Session for Dick Marsh... Maybe have something from Database group?

In January 2017, Richard E. Marsh passed away after a crystallographic career which spanned over 70 years. His work on identifying incorrect structures has had a tremendous impact on chemical crystallography, and has led to the development of structure validation tools such CheckCIF. This session celebrates the life and scientific achievements of R. Marsh. Presentations will include both personal reminiscences and scientific talks on topics inspired by Marsh's work.

Organizers:

Louise Dawe: Idawe@wlu.ca

Mike Takase:mktakase@caltech.edu

Paul Boyle: pboyle@uwo.ca

Alexander Filatov: afilatov@uchicago.edu 